\title{
Beyond Tamaki-Sato Style Unfold/Fold Transformations for Normal Logic Programs *
}

\author{
Abhik Roychoudhury $^{1}$, K. Narayan Kumar ${ }^{1,2}$, C.R. Ramakrishnan ${ }^{1}$, and \\ I.V. Ramakrishnan ${ }^{1}$ \\ 1 Dept. of Computer Science, SUNY Stony Brook, Stony Brook, NY 11794, USA. \\ \{abhik, kumar, cram, ram\}@cs.sunysb.edu \\ 2 Chennai Mathematical Institute, 92 G.N. Chetty Road, Chennai, India. \\ kumar@smi .ernet.in
}

\begin{abstract}
Unfold/fold transformation systems for logic programs have been extensively investigated. Existing unfold/fold transformation systems for normal logic programs allow only Tamaki-Sato style folding using clauses from a previous program in the transformation sequence: i.e., they fold using a single, non-recursive clause. In this paper we present a transformation system that permits folding in the presence of recursion, disjunction, as well as negation. We show that the transformations are correct with respect to various semantics of negation including the well-founded model and stable model semantics.
\end{abstract}

\section{Introduction}

Unfold/fold transformation systems for logic programs have been used for automated deduction $[8,17]$, and program specialization and optimization $[2,4,10$, 15]. Normal logic programs consist of definitions of the form $A$ :- $\phi$ where $A$ is an atom and $\phi$ is a boolean formula over atoms. Unfolding replaces an occurrence of $A$ in a program with $\phi$ while folding replaces an occurrence of $\phi$ with $A$. Folding is called reversible if its effects can be undone by an unfolding, and irreversible otherwise.

Given a logic program $P$, an unfold/fold transformation system generates a sequence of programs $P=P_{0}, P_{1}, \ldots, P_{n}$, such that for all $0 \leq i<n, P_{i+1}$ is obtained from $P_{i}$ by applying one of the two transformations. Unfold/fold transformation systems are proved correct by showing that all programs in any transformation sequence $P_{0}, P_{1}, \ldots, P_{n}$ are equivalent under a suitable semantics, such as the well-founded model semantics for normal logic programs. A comprehensive survey of research on logic program transformations appears in [14].

As an illustration of unfolding/folding, consider the sequence of normal logic programs in figure 1 . In the figure, $P_{1}$ is derived from $P_{0}$ by unfolding the occurrence of $\mathrm{q}(\mathrm{X})$ in the first clause of $P_{0}$. Program $P_{2}$ is derived from $P_{1}$ by folding the literal $\mathrm{q}(\mathrm{Y})$ in the body of the second clause of $\mathrm{p} / 1$ into $\mathrm{p}(\mathrm{Y})$ by

\footnotetext{
* This work was partially supported by NSF grants CCR-9711386, CCR-9876242, CDA-9805735 and EIA-9705998.
} 
using $\mathrm{p}(\mathrm{X}):-\mathrm{q}(\mathrm{X})$ in $P_{0}$. This clause from a previous program which is used in a folding step (the clause $\mathrm{p}(\mathrm{X}):-\mathrm{q}(\mathrm{X})$ of $P_{0}$ in this case) is called the folder.

An unfold/fold transformation system for definite logic programs was first described in a seminal paper by Tamaki and Sato [20]. It allows folding using a single clause only (conjunctive folding) from the initial program. This folder clause is required to be non-recursive, but need not be present in the current program $P_{i}$. Maher [12] proposed a transformation system using only reversible folding in which the folder clause is always drawn from the current program. However, reversibility is a restrictive condition that limits the power of unfold/fold systems by disallowing many correct transformations, such as the one used to derive $P_{2}$ from $P_{1}$ in Figure 1 . Hence, there was considerable interest in developing irreversible unfold/fold transformation systems, for both definite and normal logic programs.

Existing unfold/fold transformation systems for normal logic programs [1, 13, 18, 19] are either extensions of Maher's reversible transformation system [12] or the original Tamaki-Sato system [20]. Even for definite logic programs, irreversible transformations of programs were, until recently, restricted to either folding using non-recursive clauses (see [7]) or a single recursive clause (see [9, 21]). In [16] we proposed a transformation framework for definite logic programs which generalized the above systems by permitting folding using multiple recursive clauses. Construction of such a general transformation system for normal logic programs has remained open. Below, we describe a solution to this problem.

Overview of the results: The main result of this paper is a unfold/fold transformation system that performs folding in the presence of recursion, disjunction as well as negation (see Section 2). The transformations of [16] associates counters with program clauses (a la Kanamori and Fujita [9]) to determine the applicability of fold and unfold transformations. In this paper, we extend this book-keeping to accommodate negative literals. We show that this extension is sufficient to guarantee that the resulting transformation system preserves a variety of semantics for normal logic programs, such as the well-founded model, stable model, partial stable model, and stable theory semantics. Central to this proof is the result due to Dung and Kanchanasut [6] that preserving the semantic kernel of a program is sufficient to guarantee the preservation of the different semantics for negation listed above. However, in contrast to [1] where this idea was used to prove the correctness of Tamaki-Sato style transformations, we present a two-step proof which explicitly uses the operational counterpart of

$$
\begin{array}{lll}
\mathrm{p}(\mathrm{X}):-\mathrm{q}(\mathrm{X}) . & \mathrm{p}([]) . & \mathrm{p}([]) . \\
\mathrm{q}([]) . & \mathrm{p}([\mathrm{X} \mid \mathrm{Y}]):-\neg \mathrm{r}(\mathrm{X}), \mathrm{q}(\mathrm{Y}) . & \mathrm{p}([\mathrm{X} \mid \mathrm{Y}]):-\neg \mathrm{r}(\mathrm{X}), \mathrm{p}(\mathrm{Y}) . \\
\mathrm{q}([\mathrm{X} \mid \mathrm{Y}]):-\neg \mathrm{r}(\mathrm{X}), \mathrm{q}(\mathrm{Y}) . & \mathrm{q}([]) . & \mathrm{q}([]) . \\
& \mathrm{q}([\mathrm{X} \mid \mathrm{Y}]):-\neg \mathrm{r}(\mathrm{X}), \mathrm{q}(\mathrm{Y}) . & \mathrm{q}([\mathrm{X} \mid \mathrm{Y}]):-\neg \mathrm{r}(\mathrm{X}), \mathrm{q}(\mathrm{Y}) . \\
\quad & \quad \text { Program } P_{1} & \text { Program } P_{2}
\end{array}
$$

Fig. 1. Example of an unfold/fold transformation sequence 
semantic kernels (see Section 3). In the first step of our proof, we show that the transformations preserve positive ground derivations, which are derivations of the form $A \rightsquigarrow \neg B_{1}, \neg B_{2}, \ldots, \neg B_{n}$ such that there is a proof tree rooted at $A$ with leaves labeled $\neg B_{1}$ through $\neg B_{n}$ (apart from true). We then show that preserving positive ground derivations is equivalent to preserving the semantic kernel of the program. Thus positive ground derivations are the operational analogues of semantic kernels.

This proof suggests that we can treat the negative literals in a program as atoms of new predicates defined in a different (external) module. The correctness of the transformation system is assured as long as the transformations respect module boundaries (see Section 4). This observation indicates how a transformation system originally designed for definite logic programs (such as the one we proposed in [16]) can be readily adapted for normal logic programs.

\section{The Transformation System}

Below we present our unfold and fold transformations for normal logic programs. In the following we assume familiarity with the standard notions of terms, substitutions, unification, atoms, literals. We will use the following symbols (possibly with primes and subscripts): $P$ to denote a normal logic program; $C$ and $D$ for clauses; $A, B$ to denote atoms ; $L, K$ to denote literals $; \mathcal{N}$ to denote sequence of literals and $\sigma, \theta$ for substitutions.

In any transformation sequence $P_{0}, P_{1}, \ldots, P_{n}$ we annotate each clause $C$ in program $P_{i}$ with a pair $\left(\gamma_{l_{o}}^{i}(C), \gamma_{h i}^{i}(C)\right)$ where $\gamma_{l_{o}}^{i}(C), \gamma_{h i}^{i}(C) \in \mathbb{Z}$ and $\gamma_{l_{o}}^{i}(C) \leq$ $\gamma_{h i}^{i}(C)$. Thus, $\gamma_{l o}^{i}$ and $\gamma_{h i}^{i}$ are functions from the set of clauses in program $P_{i}$ to the set of integers $\mathbb{Z}$. The transformation rules dictate the construction of $\gamma_{l o}^{i+1}$ and $\gamma_{h i}^{i+1}$ from $\gamma_{l_{o}}^{i}$ and $\gamma_{h i}^{i}$. We assume that for any clause $C$ in the initial program $P_{0}, \gamma_{l_{o}}^{0}(C)=\gamma_{h i}^{0}(C)=1$. Intuitively, $\gamma_{l_{o}}^{i}(C)$ and $\gamma_{h i}^{i}(C)$ for a clause $C$ are analogous to the Kanamori-Fujita-style counters [9]; the separation of $h i$ and $l o$ permits us to store estimates of the counter values in the presence of disjunctive folding.

Rule 1 (Unfolding) Let $C$ be a clause in $P_{i}$ and $A$ a positive literal in the body of $C$. Let $C_{1}, \ldots, C_{m}$ be the clauses in $P_{i}$ whose heads are unifiable with $A$ with most general unifiers $\sigma_{1}, \ldots, \sigma_{m}$. Let $C_{j}^{\prime}$ be the clause that is obtained by replacing $A \sigma_{j}$ by the body of $C_{j} \sigma_{j}$ in $C \sigma_{j}(1 \leq j \leq m)$.

Then, assign $P_{i+1}:=\left(P_{i}-\{C\}\right) \cup\left\{C_{1}^{\prime}, \ldots, C_{m}^{\prime}\right\}$. Set $\gamma_{l o}^{i+1}\left(C_{j}^{\prime}\right)=\gamma_{l o}^{i}(C)+$ $\gamma_{l o}^{i}\left(C_{j}\right)$ and $\gamma_{h i}^{i+1}\left(C_{j}^{\prime}\right)=\gamma_{h i}^{i}(C)+\gamma_{h i}^{i}\left(C_{j}\right)$. The annotations of all other clauses in $P_{i+1}$ are inherited from $P_{i}$.

Rule 2 (Folding) Let $\left\{C_{1}, \ldots, C_{m}\right\}$ be clauses in $P_{i}$ and $\left\{D_{1}, \ldots, D_{m}\right\}$ be clauses in $P_{j}(j \leq i)$ where $C_{l}$ denotes the clause $A:-L_{l, 1}, \ldots, L_{l, n_{l}}, L_{1}^{\prime}, \ldots, L_{n}^{\prime}$ and $D_{l}$ denotes the clause $B_{l}:-K_{l, 1}, \ldots, K_{l, n_{l}}$. Also, let

1. $\forall 1 \leq l \leq m \exists \sigma_{l} \forall 1 \leq k \leq n_{l} L_{l, k}=K_{l, k} \sigma_{l}$, where $\sigma_{l}$ is a substitution.

2. $B_{1} \sigma_{1}=B_{2} \sigma_{2}=\ldots=B_{m} \sigma_{m}=B$ 
3. $D_{1}, \ldots, D_{m}$ are the only clauses in $P_{j}$ whose heads are unifiable with $B$.

4. $\forall 1 \leq l \leq m \sigma_{l}$ substitutes the internal variables of $D_{l}$ to distinct variables which do not appear in $\left\{A, B, L_{1}^{\prime}, \ldots, L_{n}^{\prime}\right\}$.

5. $\forall 1 \leq l \leq m \gamma_{h i}^{j}\left(D_{l}\right)<\gamma_{l o}^{i}\left(C_{l}\right)+$ Number of positive literals in the sequence $L_{1}^{\prime}, \ldots, L_{n}^{\prime}$.

Then, assign $P_{i+1}:=\left(P_{i}-\left\{C_{1}, \ldots, C_{m}\right\}\right) \cup\left\{C^{\prime}\right\}$ where $C^{\prime} \equiv A:-B, L_{1}^{\prime}, \ldots, L_{n}^{\prime}$. Set $\gamma_{l o}^{i+1}\left(C^{\prime}\right)=\min _{1 \leq l \leq m}\left(\gamma_{l o}^{i}\left(C_{l}\right)-\gamma_{h i}^{j}\left(D_{l}\right)\right)$ and $\gamma_{h i}^{i+1}\left(C^{\prime}\right)=\max _{1 \leq l \leq m}\left(\gamma_{h i}^{i}\left(C_{l}\right)-\right.$ $\left.\gamma_{l o}^{j}\left(D_{l}\right)\right)$. The annotations of all other clauses in $P_{i+1}$ are inherited from $P_{i}$.

An Example: The following example (derived from [7]) illustrates the use of our basic unfold/fold transformation system.

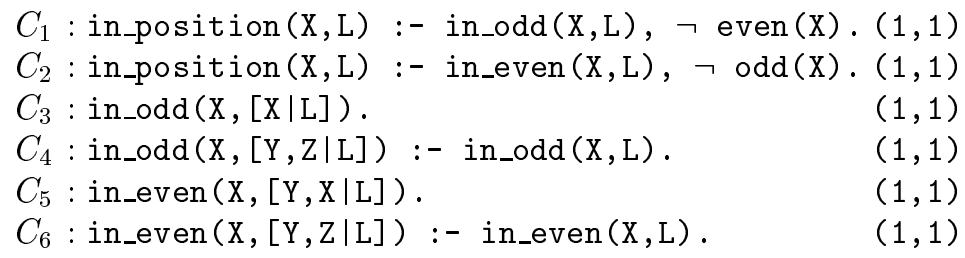

In the above program, in_odd $(X, L)$ (in_even $(X, L)$ ) is true if $X$ appears in an odd (even) position in list L. Thus, in_position $(\mathrm{X}, \mathrm{L})$ is true if $\mathrm{X}$ is in an odd (even) position in list $\mathrm{L}$, and $\mathrm{X}$ is not an even (odd) number. The odd/1 and even $/ 1$ predicates are encoded in the usual way and are not shown.

Unfolding in_odd $(\mathrm{X}, \mathrm{L})$ in $C_{1}$ we get the following clauses:

$$
\begin{aligned}
& C_{7} \text { : in_position }(\mathrm{X},[\mathrm{X} \mid \mathrm{L}]):-\neg \operatorname{even}(\mathrm{X}) \text {. }(2,2) \\
& C_{8} \text { : in_position }(\mathrm{X},[\mathrm{Y}, \mathrm{Z} \mid \mathrm{L}]) \text { : - in_odd }(\mathrm{X}, \mathrm{L}), \neg \operatorname{even}(\mathrm{X}) .(2,2)
\end{aligned}
$$

Unfolding in_even $(\mathrm{X}, \mathrm{L})$ in $C_{2}$ yields the following clauses:

$C_{9}$ : in_position $(\mathrm{X},[\mathrm{Y}, \mathrm{X} \mid \mathrm{L}]):-\neg \operatorname{odd}(\mathrm{X})$.

$C_{10}$ : in_position $(\mathrm{X},[\mathrm{Y}, \mathrm{Z} \mid \mathrm{L}])$ : - in_even $(\mathrm{X}, \mathrm{L}), \neg \operatorname{odd}(\mathrm{X}) .(2,2)$

Finally, we fold clauses $\left\{C_{8}, C_{10}\right\}$ using the clauses $\left\{C_{1}, C_{2}\right\}$ from the initial program as the folder to obtain the following definition of in_position/1.

$$
\begin{array}{ll}
C_{7}: \text { in_position }(\mathrm{X},[\mathrm{X} \mid \mathrm{L}]):-\neg \operatorname{even}(\mathrm{X}) . & (2,2) \\
C_{9}: \operatorname{in} \text {-position }(\mathrm{X},[\mathrm{Y}, \mathrm{X} \mid \mathrm{L}]):-\neg \operatorname{odd}(\mathrm{X}) . & (2,2) \\
C_{11}: \text { in_position }(\mathrm{X},[\mathrm{Y}, \mathrm{Z} \mid \mathrm{L}]):-\operatorname{in} \text { _position }(\mathrm{X}, \mathrm{L}) . & (1,1)
\end{array}
$$

Note that the final step is an irreversible folding in presence of negation that uses multiple clauses as the folder. Such a folding step is neither allowed in Tamaki-Sato style transformation systems for normal logic programs $[1,18,19]$ nor in reversible transformation systems [13].

Remark: We can maintain more elaborate book-keeping information than integer counters, thereby deriving more expressive unfold/fold systems. For instance, as in the SCOUT system described in [16], we can make the counters range over use a tuple of integers, and obtain a system that is strictly more powerful than the existing Tamaki-Sato-style systems $[20,21,9,18,19,7,1]$. The construction parallels that of the SCOUT system in [16]; details are omitted. 


\section{Proof of Correctness}

In this section, we show that our unfold/fold transformation system is correct with respect to various semantics of normal logic programs. This proof proceeds in three steps. First, we introduce the notion of positive ground derivations and show that it is preserved by the transformations. Secondly, we show that preserving positive ground derivations is equivalent to preserving semantic kernel [6]. Finally, following [1], preserving semantic kernel implies that the transformation system is correct with respect to various semantics for normal logic programs including well-founded model, stable model, partial stable model, and stable theory semantics. We begin with a review of semantic kernels.

\subsection{Semantic Kernel of a Program}

Definition 1 (Quasi-Interpretation) [6,1] A quasi-interpretation of a normal logic program $P$ is a set of ground clauses of the form $A$ : $-\neg B_{1}, \ldots, \neg B_{n}$ $(n \geq 0)$ where $A, B_{1}, \ldots, B_{n}$ are ground atoms in the Herbrand Base of $P$.

Quasi-interpretations form the universe over which semantic kernels are defined. For a given normal logic program $P$, the set of all quasi-interpretations of $P$ (denoted $Q I(P)$ ) forms a complete partial order with a least element (the empty set $\phi)$ with respect to the set inclusion relation $\subseteq$.

Definition 2 Given a normal logic program $P$, let $G$ nd $(P)$ denote the set of all possible ground instantiations of all clauses of $P$. The function $S_{P}$ on quasiinterpretations of $P$ is defined as

$$
\begin{gathered}
S_{P}: Q I(P) \rightarrow Q I(P) \\
S_{P}(I)=\left\{\mathcal{R}\left(C, D_{1}, \ldots, D_{m}\right) \mid C \in G \operatorname{Gnd}(P) \wedge D_{i} \in I, 1 \leq i \leq m\right\}
\end{gathered}
$$

where, if $D_{i}(1 \leq i \leq m)$ are ground clauses

$$
A_{i}:-\neg B_{i, 1}, \ldots, \neg B_{i, n_{i}}\left(n_{i} \geq 0\right)
$$

and $A_{1}, \ldots, A_{m}(m \geq 0)$ are the only positive literals appearing in the body of ground clause $C$, then $\mathcal{R}\left(C, D_{1}, \ldots, D_{m}\right)$ is the clause obtained by resolving the positive body literals $A_{1}, \ldots, A_{m}$ in $C$ using clauses $D_{1}, \ldots, D_{m}$ respectively.

If $P$ is a definite program, then the function $S_{P}$ is identical to the logical consequence operator $T_{P}$ [11]. The semantic kernel of the program $P$ is defined in terms of $S_{P}$ as:

Definition 3 (Semantic Kernel) [6,1] The semantic kernel of a normal logic program $P$, denoted by $S K(P)$, is the least fixed point of the function $S_{P}$, i.e.,

$$
S K(P)=\bigcup_{n \in \omega} S K^{n}(P) \text { where } S K^{0}(P)=\phi \text { and } S K^{n+1}(P)=S_{P}\left(S K^{n}(P)\right)
$$

Example : Consider the following normal logic program $P$ : 


$$
\begin{aligned}
& \mathrm{p}:-\neg \mathrm{q}, \mathrm{r} . \\
& \mathrm{r}:-\neg \mathrm{r} .
\end{aligned}
$$

The semantic kernel of $P$ will be computed as follows.

$$
\begin{aligned}
& S K^{0}(P)=\{\} \\
& S K^{1}(P)=S_{P}\left(S K^{0}(P)\right)=\{(\mathrm{r}:-\neg \mathrm{r})\} \\
& S K^{2}(P)=S_{P}\left(S K^{1}(P)\right)=\{(\mathrm{r}:-\neg \mathrm{r}),(\mathrm{p}:-\neg \mathrm{q}, \neg \mathrm{r})\} \\
& S K^{3}(P)=S_{P}\left(S K^{2}(P)\right)=S K^{2}(P) \\
& \text { Therefore, } S K(P)=\{(\mathrm{r}:-\neg \mathrm{r}),(\mathrm{p}:-\neg \mathrm{q}, \neg \mathrm{r})\}
\end{aligned}
$$

The following theorem from [1] formally states the equivalence of $P$ and $S K(P)$ with respect to various semantics of normal logic programs.

Theorem 1 [Aravindan and Dung] Let $P$ be a normal logic program and $S K(P)$ be its semantic kernel. Then:

(1) If $P$ is a definite logic program, then $P$ and $S K(P)$ have the same least Herbrand Model.

(2) If $P$ is a stratified program, then $P$ and $S K(P)$ have the same perfect model semantics.

(3) $P$ and $S K(P)$ have the same well-founded model.

(4) $P$ and $S K(P)$ have the same stable model(s).

(5) $P$ and $S K(P)$ have the same set of partial stable models.

(6) $P$ and $S K(P)$ have the same stable theory semantics.

\subsection{Preserving the Semantic Kernel}

We now show that in any transformation sequence $P_{0}, P_{1}, \ldots, P_{n}$ where $\forall 0 \leq$ $i<n P_{i+1}$ is obtained from $P_{i}$ by applying unfolding (rule 1) or folding (rule 2), the semantic kernel is preserved, i.e., $S K\left(P_{0}\right)=S K\left(P_{1}\right)=\ldots=S K\left(P_{n}\right)$. To do so, we introduce the following notion of a positive ground derivation:

Definition 4 (Positive ground derivation) A positive ground derivation of a literal in a normal logic program $P$ is a tree $T$ such that: (1) each internal node of $T$ is labeled with a ground atom (2) each leaf node of $T$ is labeled with a negative ground literal or the special symbol true, and (3) for any internal node $A$ of $T, A:-L_{1}, \ldots, L_{n}$ must be a ground instance of a clause in program $P$ where $L_{1}, \ldots, L_{n}$ are the children of $A$ in $T$.

Thus, consider any positive ground derivation $T$ in program $P$. Let the root of $T$ be labeled with the ground literal $L$ and let $\mathcal{N}$ be the sequence of negative literals derived in $T$, i.e., $\mathcal{N}$ is formed by appending the negative literals appearing in the leaf nodes of $T$ from left to right. Then we say that $L$ derives $\mathcal{N}$ in $P$, and denote such derivations by $L \rightsquigarrow P \mathcal{N}$ (and $L \rightsquigarrow \mathcal{N}$ if $P$ is obvious from the context). We overload this notation, often denoting existence of such derivations also by $L \rightsquigarrow P \mathcal{N}$. Note that if $L$ is a ground negative literal, there is only one positive ground derivation for $L$ in any program, namely the empty derivation $L \rightsquigarrow L$. We now define: 
Definition 5 (Weight of a positive ground derivation) Let $L \rightsquigarrow P \mathcal{N}$ be a positive ground derivation. The number of internal nodes in this derivation (i.e. the number of nodes labeled with a ground positive literal) is called the weight of the derivation.

Definition 6 (Weight of a pair) Let $P_{0}, P_{1}, \ldots, P_{n}$ be a transformation sequence of normal logic programs. Let $L$ be a ground literal, $\mathcal{N}$ be a (possibly empty) sequence of ground negative literals s.t. $L \rightsquigarrow P_{0} \mathcal{N}$. Then, the weight of $(L, \mathcal{N})$, denoted by $w(L, \mathcal{N})$, is the minimum of the weights of positive ground derivations of the form $L \rightsquigarrow P_{0} \mathcal{N}$.

Note that for any program $P_{i}$ in the transformation sequence, the weight of any pair $w(L, \mathcal{N})$ is defined as the weight of the smallest derivation $L \rightsquigarrow P_{0} \mathcal{N}$.

Definition 7 Let $P_{0}, P_{1}, \ldots, P_{n}$ be a transformation sequence of normal logic programs. A positive ground derivation $L \rightsquigarrow P_{i} \mathcal{N}$ is said to be weakly weightconsistent if for every ground instance $A:-L_{1}, \ldots, L_{k}$ of a clause $C$ used in this derivation, we have $w\left(A, \mathcal{N}_{A}\right) \leq \gamma_{h i}^{i}(C)+\sum_{1<l<k} w\left(L_{l}, \mathcal{N}_{l}\right)$ where $\mathcal{N}_{A}, \mathcal{N}_{1}, \ldots, \mathcal{N}_{k}$ are the sequence of negative literals derived from $A, L_{1}, \ldots, L_{k}$ in this derivation.

Definition 8 Let $P_{0}, P_{1}, \ldots, P_{n}$ be a transformation sequence of normal logic programs. A positive ground derivation $L \rightsquigarrow P_{i} \mathcal{N}$ is said to be strongly weightconsistent if for every ground instance $A:-L_{1}, \ldots, L_{k}$ of a clause $C$ used in this derivation, we have

$-w\left(A, \mathcal{N}_{A}\right) \geq \gamma_{l o}^{i}(C)+\sum_{1<l<k} w\left(L_{l}, \mathcal{N}_{l}\right)$

$-\forall 1 \leq l \leq k w\left(A, \mathcal{N}_{A}\right)>w\left(L_{l}, \mathcal{N}_{l}\right)$

where $\mathcal{N}_{A}, \mathcal{N}_{1}, \ldots, \mathcal{N}_{k}$ are the negative literal sequences derived from $A, L_{1}, \ldots, L_{k}$ in this derivation.

Definition 9 (Weight consistent program) Let $P_{0}, P_{1}, \ldots, P_{n}$ be a transformation sequence of normal logic programs. Then, program $P_{i}$ is said to be weight consistent if

- for any pair $(L, \mathcal{N})$, whenever $L$ derives $\mathcal{N}$ in $P_{i}$, there is a strongly weight consistent positive ground derivation $L \rightsquigarrow P_{i} \mathcal{N}$.

- every positive ground derivation in $P_{i}$ is weakly weight consistent.

Using the above definitions, we now state certain invariants which always hold after the application of any unfold/fold transformation.

- I1 $\left(P_{i}\right) \equiv \forall L \forall \mathcal{N}\left(L\right.$ derives $\mathcal{N}$ in $P_{0} \Leftrightarrow L$ derives $\mathcal{N}$ in $\left.P_{i}\right)$.

- I2( $\left.P_{i}\right) \equiv P_{i}$ is a weight consistent program

We now show that these invariants are maintained after every unfolding and folding step. This allows us to claim that the set of positive ground derivations of $P_{0}$ is identical to the set of positive ground derivations of program $P_{i}$. 
Lemma 1 If $\left(\forall j \leq i\right.$ II $\left.\left(P_{j}\right)\right)$ holds, then $\forall L \forall \mathcal{N}\left(L\right.$ derives $\mathcal{N}$ in $P_{i+1} \Rightarrow L$ derives $\mathcal{N}$ in $P_{i}$ )

Lemma 2 (Preserving Weak Weight Consistency) Let $P_{0}, \ldots, P_{i}, P_{i+1}$ be an unfold/fold transformation sequence s.t. $\forall 0 \leq j \leq i I 1\left(P_{j}\right) \wedge I 2\left(P_{j}\right)$. Then, all positive ground derivations of $P_{i+1}$ are weakly weight consistent.

The proofs for both Lemma 1 and 2 follow by induction on the weight of positive ground derivations in $P_{i+1}$. We now establish the main theorem concerning the preservation of positive ground derivations in a transformation sequence.

Theorem 2 Let $P_{0}, P_{1}, \ldots$ be a sequence of normal logic programs where $P_{i+1}$ is obtained from $P_{i}$ by applying unfolding (rule 1) or folding (rule 2). Then $\forall i \geq 0 I 1\left(P_{i}\right) \wedge I 2\left(P_{i}\right)$.

Proof : The proof proceeds by induction on $i$. For the base case, $I 1\left(P_{0}\right)$ holds trivially, and $I 2\left(P_{0}\right)$ holds because every positive ground derivation of $P_{0}$ is weakly weight consistent, and for any pair $(L, \mathcal{N})$ the smallest positive ground derivation $L \rightsquigarrow P_{0} \mathcal{N}$ is strongly weight consistent.

For the induction step, we need to show $I 1\left(P_{i+1}\right) \wedge I 2\left(P_{i+1}\right)$. By Lemma 1 we have $L \rightsquigarrow P_{i+1} \mathcal{N} \Rightarrow L \rightsquigarrow P_{i} \mathcal{N}$, and by Lemma 2 we know that all positive ground derivations of $P_{i+1}$ are weakly weight consistent. We need to show that (i) $L \rightsquigarrow P_{i} \mathcal{N} \Rightarrow L \rightsquigarrow P_{i+1} \mathcal{N}$, and (ii) for any pair $(L, \mathcal{N})$ s.t. $L \rightsquigarrow P_{i+1} \mathcal{N}$, there exists a strongly weight consistent derivation $L \rightsquigarrow \mathcal{N}$ in $P_{i+1}$. Thus, it suffices to prove that for any pair $(L, \mathcal{N})$ s.t $L \rightsquigarrow P_{i} \mathcal{N}$, there exists a strongly weight consistent derivation $L \rightsquigarrow P_{i+1} \mathcal{N}$.

Consider a pair $(L, \mathcal{N})$ such that $L \rightsquigarrow P_{i} \mathcal{N}$. Since $P_{i}$ is weight consistent, therefore there exists a strongly weight consistent derivation $L \rightsquigarrow \mathcal{N}$ in $P_{i}$. Let this be called $D r$. We now construct a strongly weight consistent derivation $D r^{\prime} \equiv L \rightsquigarrow P_{i+1} \mathcal{N}$. Construction of $D r^{\prime}$ proceeds by induction on the weight of $(L, \mathcal{N})$ pairs. The base case occurs when $L$ is a negative literal, $\mathcal{N}=L$ and $w(L, \mathcal{N})=0$. We then trivially have the same derivation $L \rightsquigarrow \mathcal{N}$ in $P_{i+1}$ as well. Otherwise if $L$ is a positive literal, let $C$ be the clause used at the root of $D r$. Let $L:-L_{1}, \ldots, L_{n}$ be the ground instantiation of $C$ used at the root of $D r$. Since $D r$ is strongly weight consistent $w(L, \mathcal{N})>w\left(L_{l}, \mathcal{N}_{l}\right)$ where $\mathcal{N}_{l}$ is the sequence of negative literals derived by $L_{l}$ for all $1 \leq l \leq n$. Hence, we have strongly weight consistent derivations $L_{l} \rightsquigarrow P_{i+1} \mathcal{N}_{l}$. We construct $D r^{\prime}$ by considering the following cases :

Case 1: $C$ is inherited from $P_{i}$ to $P_{i+1}$

$D r^{\prime}$ is constructed with the clause $L:-L_{1}, \ldots, L_{n}$ at the root and then appending the derivations $L_{l} \rightsquigarrow P_{i+1} \mathcal{N}_{l}$ for all $1 \leq l \leq n$. This derivation $D r^{\prime}$ is strongly weight consistent.

Case 2: $C$ is unfolded. 
Let the $L_{1}$ be the positive body literal of $C$ that is unfolded. Let the clause used to resolve $L_{1}$ in the derivation $D r$ be $C_{1}$ and the ground instance of $C_{1}$ used be $L_{1}:-L_{1,1}, \ldots, L_{1, k}$. By definition of unfolding $L:-L_{1,1}, \ldots, L_{1, k}, L_{2}, \ldots, L_{n}$ is a ground instance of a clause $C_{1}^{\prime}$ in $P_{i+1}$ with $\gamma_{l o}^{i+1}\left(C_{1}^{\prime}\right)=\gamma_{l o}^{i}(C)+\gamma_{l o}^{i}\left(C_{1}\right)$. Also, let $\mathcal{N}_{1,1}, \ldots, \mathcal{N}_{1, k}$ be the sequence of negative literals derived by $L_{1,1}, \ldots, L_{1, k}$ in $D r$. Then, by strong weight consistency $w\left(L_{1, l}, \mathcal{N}_{1, l}\right)<w\left(L_{1}, \mathcal{N}_{1}\right)<w(L, \mathcal{N})$ for all $1 \leq l \leq k$. Thus we have strongly weight consistent derivations $L_{1, l} \rightsquigarrow P_{i+1}$ $\mathcal{N}_{1, l}$. We construct $D r^{\prime}$ by applying $L:-L_{1,1}, \ldots, L_{1, k}, L_{2}, \ldots, L_{n}$ at the root and then appending the strongly weight consistent derivations $L_{1, l} \rightsquigarrow P_{i+1} \mathcal{N}_{1, l}$ (for all $1 \leq l \leq k$ ) and $L_{l} \rightsquigarrow P_{i+1} \mathcal{N}_{l}$ (for all $2 \leq l \leq n$ ). Since $D r$ is strongly weight consistent, therefore

$$
\begin{array}{ll}
\quad w(L, \mathcal{N}) \geq \gamma_{l o}^{i}(C)+\sum_{1 \leq l \leq n} w\left(L_{l}, \mathcal{N}_{l}\right) \\
\text { and } \quad w\left(L_{1}, \mathcal{N}_{1}\right) \geq \gamma_{l o}^{i}\left(C_{1}\right)+\sum_{1 \leq l \leq k} w\left(L_{1, l}, \mathcal{N}_{1, l}\right) \\
\Rightarrow \quad w(L, \mathcal{N}) \geq \gamma_{l o}^{i+1}\left(C_{1}^{\prime}\right)+\sum_{1 \leq l \leq k} w\left(L_{1, l}, \mathcal{N}_{1, l}\right)+\sum_{2 \leq l \leq n} w\left(L_{l}, \mathcal{N}_{l}\right)
\end{array}
$$

This shows that $D r^{\prime}$ is strongly weight consistent.

Case 3: $C$ is folded

Let $C$ (potentially with other clauses) be folded, using folder clause(s) from $P_{j}(j \leq i)$, to clause $C^{\prime}$ in $P_{i+1}$. Assume that $L_{1}, \ldots, L_{k}$ are the instances of the body literals of $C$ which are folded. Then, $C^{\prime}$ must have a ground instance of the form $L:-B, L_{k+1}, \ldots, L_{n}$, where $B:-L_{1}, \ldots, L_{k}$ is a ground instance of a folder clause $D$ in $P_{j}$. Since, we have derivations $L_{l} \rightsquigarrow P_{i} \mathcal{N}_{l}$ for all $1 \leq l \leq k$, therefore by $I 1\left(P_{i}\right) \wedge I 1\left(P_{j}\right)$ there exist derivations $L_{l} \rightsquigarrow P_{j} \mathcal{N}_{l}$. Then, there exists a derivation $B \rightsquigarrow P_{j} \mathcal{N}_{B}$ where $\mathcal{N}_{B}$ is obtained by appending the sequences $\mathcal{N}_{1}, \ldots, \mathcal{N}_{k}$. Since $P_{j}$ is a weight consistent program, this derivation must be weakly weight consistent, and therefore $w\left(B, \mathcal{N}_{B}\right) \leq \gamma_{h i}^{j}(D)+\sum_{1 \leq l \leq k} w\left(L_{l}, \mathcal{N}_{l}\right)$. By strong weight consistency of $D r$, we have

$$
\begin{aligned}
w(L, \mathcal{N}) & \geq \gamma_{l o}^{i}(C)+\sum_{1 \leq l \leq k} w\left(L_{l}, \mathcal{N}_{l}\right)+\sum_{k+1 \leq l \leq n} w\left(L_{l}, \mathcal{N}_{l}\right) \\
& \geq \gamma_{l o}^{i}(C)+w\left(B, \mathcal{N}_{B}\right)-\gamma_{h i}^{j}(D)+\sum_{k+1 \leq l \leq n} w\left(L_{l}, \mathcal{N}_{l}\right) \\
& >w\left(B, \mathcal{N}_{B}\right) \text { (by condition (5) of folding) }
\end{aligned}
$$

Hence there exists a strongly weight consistent derivation $B \rightsquigarrow P_{i+1} \mathcal{N}_{B}$. We now construct $D r^{\prime}$ with $L:-B, L_{k+1}, \ldots, L_{n}$ at the root and then appending below the strongly weight consistent derivations $B \rightsquigarrow P_{i+1} \mathcal{N}_{B}, L_{k+1} \rightsquigarrow P_{i+1}$ $\mathcal{N}_{k+1}, \ldots, L_{n} \rightsquigarrow P_{i+1} \mathcal{N}_{n}$. To show that $D r^{\prime}$ is strongly weight consistent, note that $\gamma_{l o}^{i+1}\left(C^{\prime}\right) \leq \gamma_{l o}^{i}(C)-\gamma_{h i}^{j}(D)$ since $C$ and $D$ are folded and folder clauses. Combining this with $\left(^{*}\right)$,

$$
w(L, \mathcal{N}) \geq \gamma_{l o}^{i+1}\left(C^{\prime}\right)+w\left(B, \mathcal{N}_{B}\right)+\sum_{k+1 \leq l \leq n} w\left(L_{l}, \mathcal{N}_{l}\right)
$$


This completes the proof.

Thus we have shown that all positive ground derivations are preserved at every step of our transformation. Now we show how our notion of positive ground derivations directly corresponds to the notion of semantic kernel. Intuitively, this connection is clear, since a clause in the semantic kernel of program $P$ is derived by repeatedly resolving the positive body literals of a ground instance of a clause in $P$ until the body contains only negative literals. Formally, we prove that :

Theorem 3 Let $P$ be a normal logic program and $A, B_{1}, \ldots, B_{n}(n \geq 0)$ be ground atoms in the Herbrand base of $P$. Let $\mathcal{N}$ be the sequence $\neg B_{1}, \ldots, \neg B_{n}$. Then, $A$ derives $\mathcal{N}$ in $P$ iff $(A:-\mathcal{N}) \in S K(P)$

Proof Sketch: We prove $A \rightsquigarrow P \mathcal{N} \Rightarrow(A:-\mathcal{N}) \in S K(P)$ by strong induction on the weight (i.e. the number of internal nodes, refer definition 5$)$ in the derivation $A \rightsquigarrow P \mathcal{N}$. The proof for $(A:-\mathcal{N}) \in S K(P) \Rightarrow A \rightsquigarrow P \mathcal{N}$ follows by fixed-point induction.

We can now prove that the semantic kernel is preserved across any unfold/fold transformation sequence.

Corollary 3 (Preservation of Semantic Kernel) Suppose $P_{0}, \ldots, P_{n}$ is a sequence of normal logic programs where $P_{i+1}$ is obtained from $P_{i}$ by unfolding (Rule 1) or folding (Rule 2). Then $\forall 0 \leq i<n S K\left(P_{i}\right)=S K\left(P_{0}\right)$.

Proof: We prove that $S K\left(P_{0}\right)=S K\left(P_{i}\right)$ for any arbitrary $i$. By Theorem 2 we know that $A \rightsquigarrow P_{0} \mathcal{N} \Leftrightarrow A \rightsquigarrow P_{i} \mathcal{N}$ for any ground atom $A$ and sequence of ground negative literals $\mathcal{N}$. Then, using Theorem 3 we get $(A:-\mathcal{N}) \in S K\left(P_{0}\right) \Leftrightarrow$ $(A:-\mathcal{N}) \in S K\left(P_{i}\right)$. Thus, $S K\left(P_{0}\right)=S K\left(P_{i}\right)$.

Following Theorem 1 and Corollary 3 we have:

Theorem 4 (Correctness of Unfolding/Folding) Let $P_{0}, \ldots, P_{n}$ be a sequence of normal logic programs where $P_{i+1}$ is obtained from $P_{i}$ by an application of unfolding (Rule 1) or folding (Rule 2). Then, for all $0 \leq i<n$ we have

(1) If $P_{0}$ is a definite logic program, then $P_{0}$ and $P_{i}$ have the same least Herbrand Model.

(2) If $P_{0}$ is a stratified program, then $P_{0}$ and $P_{i}$ have the same perfect model semantics.

(3) $P_{0}$ and $P_{i}$ have the same well-founded model.

(4) $P_{0}$ and $P_{i}$ have the same stable model(s).

(5) $P_{0}$ and $P_{i}$ have the same set of partial stable models.

(6) $P_{0}$ and $P_{i}$ have the same stable theory semantics.

\section{Discussion}

In this paper we have presented an unfold/fold transformation system, which to the best of our knowledge, is the first to permit folding in the presence of recursion, disjunction, as well as negation. Such a system is particularly important for verifying temporal properties of parameterized concurrent systems (such as 
a $n$-bit shift register for any $n$ ) using logic program evaluation and deduction [5, 17].

The transformation system presented in this paper can be extended to incorporate a goal replacement rule which allows the replacement of a conjunction of atoms in the body of a clause with another semantically equivalent conjunction of atoms provided certain conditions are satisfied (which ensure preservation of weight consistency). In future, it would be interesting to study how we can perform multiple replacements simultaneously without compromising correctness (as discussed in [3]).

Apart from the transformation system, the details of the underlying correctness proof reveal certain interesting aspects generic to such transformation systems. First of all, our proof exploits a degree of modularity that is inherent in the unfold/fold transformations for logic programs. Consider a modular decomposition of a definite logic program where each predicate is fully defined in a single module. Each module has a set of "local" predicates defined in the current module and a set of "external" predicates used (and not defined) in the current module. It is easy to see that Lemma 1, 2 and Theorem 2 can be modified to show that unfold/fold transformations preserve the set of local ground derivations of a program. We say that $A \rightsquigarrow B_{1}, B_{2}, \ldots, B_{n}$ is a local ground derivation (analogous to a positive ground derivation), if each $B_{i}$ contains an external predicate, and there is a proof tree rooted at $A$ whose leaves are labeled with $B_{1}, \ldots, B_{n}$ (apart from true). Consequently, transformations of a normal logic program $P$, can be simulated by an equivalent positive program module $Q$ obtained by replacing negative literals in $P$ with new positive external literals. The newly introduced literals can be appropriately defined in a separate module. Thus any transformation system for definite logic programs that preserves local ground derivations also preserves the semantic kernels of normal logic programs.

Secondly, we showed that positive ground derivations form the operational counterpart to semantic kernels. This result, which makes explicit an idea in the proof of Aravindan and Dung [1], enables the correctness proof to be completed by connecting the other two steps: an operational first step, where the measure consistency technique is used to show the preservation of positive ground derivations and the final model-theoretic step that applies the results of Dung and Kanchanasut [6] relating semantic kernels to various semantics for normal logic programs.

Semantic kernel is a fundamental concept in the study of model-based semantics. By it very nature, however, semantic kernels cannot be used in proving operational equivalences such as finite failure and computed answer sets. The important task then is to formulate a suitable operational notion that plays the role of semantic kernel in the correctness proofs with respect to these equivalences.

\section{References}

1. C. Aravindan and P.M. Dung. On the correctness of unfold/fold transformations of normal and extended logic programs. Journal of Logic Programming, pages 295-322, 1995. 
2. A. Bossi, N. Cocco, and S. Dulli. A method of specializing logic programs. $A C M$ TOPLAS, pages 253-302, 1990.

3. A. Bossi, N. Cocco, and S. Etalle. Simultaneous replacement in normal programs. Journal of Logic and Computation, 6(1):79-120, February 1996.

4. D. Boulanger and M. Bruynooghe. Deriving unfold/fold transformations of logic programs using extended OLDT-based abstract interpretation. Journal of Symbolic Computation, pages 495-521, 1993.

5. B. Cui, Y. Dong, X. Du, K. Narayan Kumar, C.R. Ramakrishnan, I.V. Ramakrishnan, A. Roychoudhury, S.A. Smolka, and D.S. Warren. Logic programming and model checking. In Proceedings of PLILP/ALP, LNCS 1490, pages 1-20, 1998.

6. P.M. Dung and K. Kanchanasut. A fixpoint approach to declarative semantics of logic programs. Proceedings of North American Conference on Logic Programming, 1:604-625, 1989.

7. M. Gergatsoulis and M. Katzouraki. Unfold/fold transformations for definite clause programs. In Proceedings of PLILP, LNCS 844, pages 340-354, 1994.

8. T. Kanamori and H. Fujita. Formulation of Induction Formulas in Verification of Prolog Programs. Proceedings of International Conference on Automated Deduction (CADE), pages 281-299, 1986.

9. T. Kanamori and H. Fujita. Unfold/fold transformation of logic programs with counters. In USA-Japan Seminar on Logics of Programs, 1987.

10. M. Leuschel, D. De Schreye, and A. De Waal. A conceptual embedding of folding into partial deduction : Towards a maximal integration. In Joint International Conference and Symposium on Logic Programming, pages 319-332, 1996.

11. J.W. Lloyd. Foundations of Logic Programming, 2nd Ed. Springer-Verlag, 1993.

12. M. J. Maher. Correctness of a logic program transformation system. Technical report, IBM T.J. Watson Research Center, 1987.

13. M. J. Maher. A transformation system for deductive database modules with perfect model semantics. Theoretical Computer Science, 110:377-403, 1993.

14. A. Pettorossi and M. Proietti. Transformation of logic programs, volume 5 of Handbook of Logic in Artificial Intelligence, pages 697-787. Oxford University Press, 1998.

15. A. Pettorossi, M. Proietti, and S. Renault. Reducing nondeterminism while specializing logic programs. In Proceedings of POPL, pages 414-427, 1997.

16. A. Roychoudhury, K. Narayan Kumar, C.R. Ramakrishnan, and I.V. Ramakrishnan. A parameterized unfold/fold transformation framework for definite logic programs. In Principles and Practice of Declarative Programming (PPDP), LNCS 1702, pages 396-413, 1999.

17. A. Roychoudhury, K. Narayan Kumar, C.R. Ramakrishnan, and I.V. Ramakrishnan. Proofs by program transformations. To appear in proceedings of Logic-based Program Synthesis and Transformation (LOPSTR), 1999.

18. H. Seki. Unfold/fold transformation of stratified programs. In Theoretical Computer Science, pages 107-139, 1991.

19. H. Seki. Unfold/fold transformation of general logic programs for well-founded semantics. In Journal of Logic Programming, pages 5-23, 1993.

20. H. Tamaki and T. Sato. Unfold/fold transformations of logic programs. In Proceedings of International Conference on Logic Programming, pages 127-138, 1984.

21. H. Tamaki and T. Sato. A generalized correctness proof of the unfold/ fold logic program transformation. Technical report, Ibaraki University, Japan, 1986. 